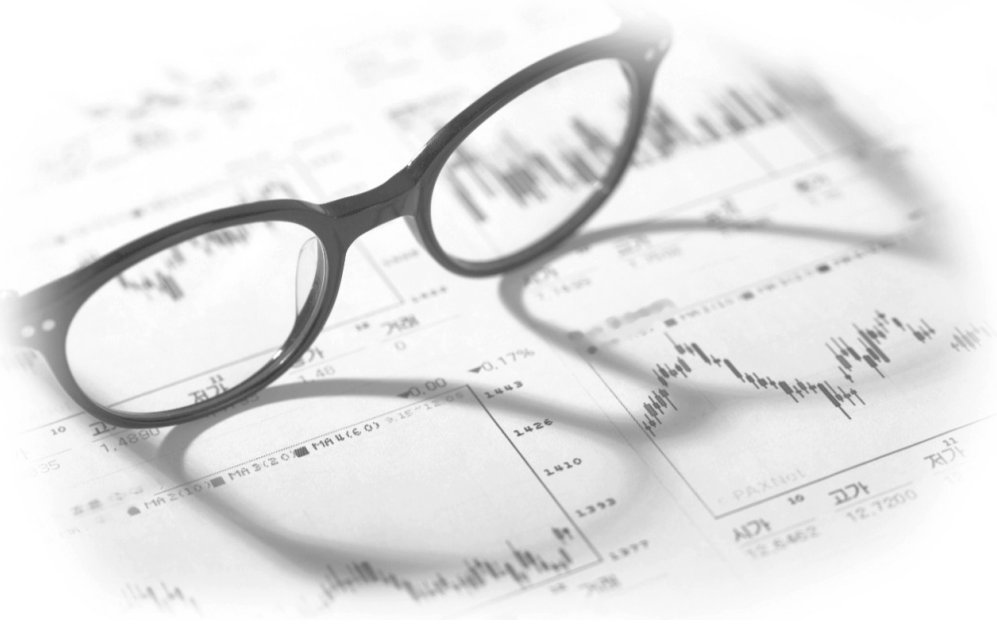

BLOOD RESEARCH

Volume $54 \cdot$ Number $3 \cdot$ September 2019

https://doi.org/10.5045/br.2019.54.3.159

Perspective

\section{Optimizing carfilzomib use in multiple myeloma treatment}

\author{
Sung-Soo Yoon, M.D., Ph.D. \\ Department of Internal Medicine, Seoul National University \\ College of Medicine, Seoul National University Hospital, Seoul, \\ Korea
}

Received on June 4, 2019; Revised on June 13, 2019;

Accepted on June 14, 2019

\section{Introduction}

The treatment landscape of multiple myeloma (MM) continues to evolve. More liberal use of various proteasome inhibitors and immunomodulatory agents is being actualized in Korea, making continuous optimization of MM treatment possible. Amidst it all, the biggest issue in year 2018 was perhaps the insurance coverage of carfilzomib use as second-line treatment, either with dexamethasone or lenalidomide plus dexamethasone. One year into carfilzomib use, we are met with notable improvements and new challenges. Here, I would like to share my center's experience (Fig. 1) with carfilzomib and address some of the issues we are faced with.

\section{Current Issues}

\section{How tolerable and efficacious is second-line carfilzomib in the real world?}

For autologous stem cell transplantation (ASCT)-eligible patients, there is little concern about choosing carfilzomib- based triplet therapy as second-line treatment. On the other hand, for ASCT-ineligible patients, the decision is more complicated. We have already determined from the ASPIRE trial [1-4] that carfilzomib, lenalidomide, and dexamethasone $(\mathrm{KRd})$ is superior to lenalidomide and dexamethasone ( $\mathrm{Rd})$, regardless of prior exposure to bortezomib, time to relapse, and age. However, how readily these results can be implemented in real-world practice is a different matter because we still have safety concerns, especially regarding cardiac toxicities and the tolerability of Korean elderly patients to both the schedule and dose of KRd. For answers, we compared the outcomes of patients with MM $\geq 65$ years old who received either $\mathrm{Rd}$ or $\mathrm{KRd}$ as second-line treatment after bortezomib, melphalan, and prednisone since January 2016. As shown in Table 1, 26 patients received Rd, 14 patients received $\mathrm{KRd}$, and there were no differences in baseline characteristics between the 2 groups. During the median follow-up of 35 months, the median progression-free

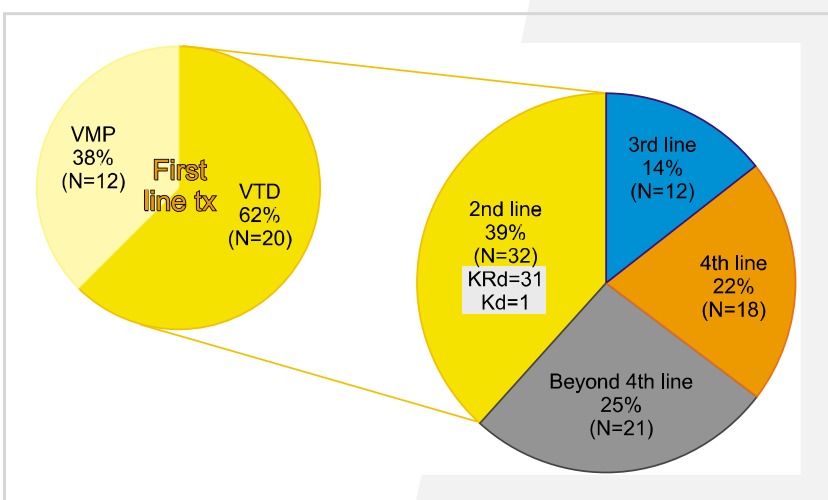

Fig. 1. Overview of carfilzomib use at Seoul National University Hospital since 2016.

Abbreviations: KRd, carfilzomib, lenalidomide, dexamethasone; Rd, lenalidomide, dexamethasone; $\mathrm{tx}$, treatment; VMP, bortezomib, melphalan, prednisone; VTD, bortezomib, thalidomide, dexamethasone. 
Table 1. Characteristics and responses among patients $\geq 65$ years old.

\begin{tabular}{lccl}
\hline & Total & Rd & \multicolumn{1}{c}{ KRd } \\
\hline $\mathrm{N}$ & 40 & 26 & \multicolumn{1}{c}{14} \\
$\begin{array}{c}\text { Age, yr (median, } \\
\text { range) }\end{array}$ & $70(65-81)$ & $70(65-81)$ & $69(65-78)$ \\
Sex (male, \%) & $24(60 \%)$ & $15(57.7 \%)$ & $9(64.3 \%)$ \\
HR by FISH ${ }^{\text {a) }}$ & $7(17.5 \%)$ & $3(11.5 \%)$ & $4(28.6 \%)$ \\
N of cycles & $\mathrm{NA}$ & $8(2-43)$ & $3(2-17)$ \\
$\quad($ median, range) & & & \\
Best response & & & \\
$\quad$ CR & $13(32.5 \%)$ & $9(34.6 \%)$ & $4(28.6 \%)$ \\
VGPR & $1(2.5 \%)$ & 0 & $1(7.1 \%)$ \\
PR & $14(35 \%)$ & $10(38.5 \%)$ & $7(50 \%)$ \\
SD & $9(22.5 \%)$ & $7(26.9 \%)$ & $2(14.3 \%)$ \\
\hline
\end{tabular}

${ }^{a}$ High risk defined as del17p, $\mathrm{t}(4 ; 14), \mathrm{t}(14 ; 16)$.

Abbreviations: $\mathrm{CR}$, complete response; $\mathrm{FISH}$, fluorescence in situ hybridization; $\mathrm{HR}$, high risk; KRd, carfilzomib, lenalidomide, and dexamethasone; NA, not applicable; PR, partial response; Rd, lenalidomide and dexamethasone; SD, stable disease; VGPR, very good partial response.

survival (PFS) was 10 months in both the Rd and KRd groups $(P=0.905)$. When the best response was considered, the proportion of patients showing very good partial response (VGPR) or better response was slightly higher in the KRd group (38.5\%) compared to that in the Rd group $(34.6 \%)$. If partial response (PR) was used as the cutoff, the difference became more prominent $(78.6 \%$ for $\mathrm{KRd}$ vs. $73.1 \%$ for $\mathrm{Rd}$ ). Considering that most of the patients in the KRd group are still receiving KRd, while most of the patients in the Rd group have progressed and are currently receiving third-line treatment, I expect the gap to widen in favor of KRd with longer follow-up. Furthermore, contrary to popular belief, only 1 patient from the KRd group had a significant cardiac adverse event in the form of heart failure, but even this patient recovered without sequelae. In my opinion, because we are so aware of the possible adverse events of carfilzomib at this point that we monitor our patients with vigilance and promptly intervene as necessary, unexpected events rarely occur. Of course, with these being retrospective data from a rather small number of patients, there is the innate pitfall of selection bias, and we were unable to evaluate the impact of cytogenetic/molecular risk stratification on prognosis. However, given the biology of MM, I stand by my opinion that we should become more comfortable with using KRd, even in ASCT-ineligible patients.

\section{Where does autologous stem cell transplantation fit in?}

Although ASCT remains an integral part of $\mathrm{MM}$ treatment, the procedure has failed to demonstrate a significant impact on overall survival on multiple occasions and is continuously being challenged [5-8]. We often pose

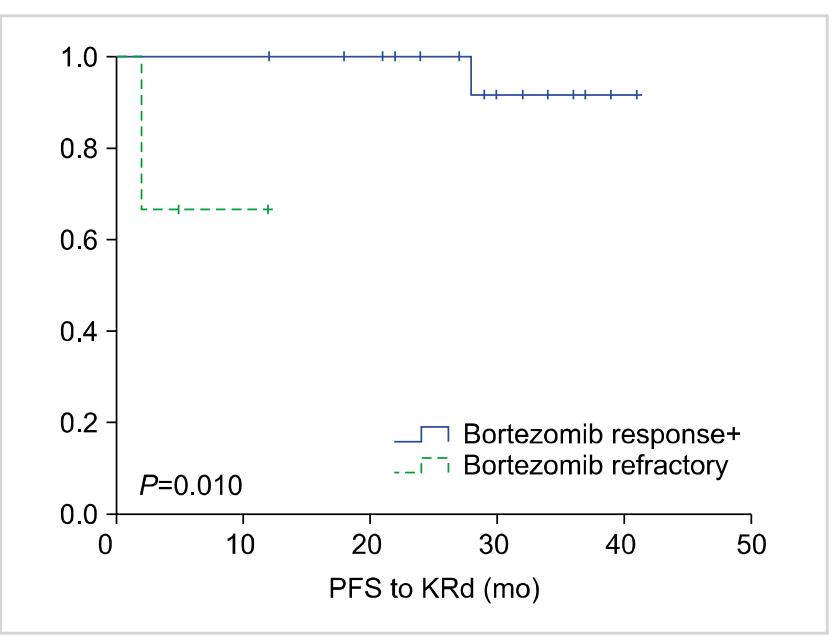

Fig. 2. PFS following KRd between bortezomib-responsive patients versus bortezomib-refractory patients.

Abbreviations: KRd, carfilzomib, lenalidomide, dexamethasone; PFS, progression-free survival.

the same question to ourselves: with all these novel agents, is ASCT still necessary? In short, the answer seems to be yes. We selected ASCT-eligible patients who received first-line bortezomib, thalidomide, and dexamethasone (VTD) and compared their response to second-line KRd according to initial bortezomib response (Fig. 2). We found that patients who were refractory to bortezomib were associated with significantly shorter PFS following KRd compared to patients who were responsive to bortezomib $(P=0.010)$. In these patients, upfront ASCT is warranted even if they seem to be responding well to KRd. Again, the number of patients is too small to draw affirmative conclusions, but based on experience at our center, we prepare our patients for ASCT after 4-5 cycles of KRd in such circumstances.

As for the second ASCT, things become hazier because the very role of the second ASCT in relapsed MM is a topic of controversy [9]. Some advocate the use of the second ASCT in patients relapsing after primary therapy that includes ASCT with an initial duration of remission of more than 18 months. Thus far, there have been 11 cases of relapse after VTD, followed by upfront ASCT, and then second-line KRd. Three patients among these proceeded onto second ASCT and achieved complete response, while the remaining 8 are still receiving KRd. Since there is no consensus on the matter, the decision for the second ASCT was based on the attending physician's discretion. Presumably, most of these patients would already harbor excess cryopreserved cells from the first ASCT; thus, collection timing with regards to lenalidomide exposure would not be such a significant issue. On a personal note, if the patient is responding well to second-line KRd in this scenario, I do not favor second ASCT, as we can administer KRd for 18 cycles followed by $\mathrm{Rd}$ for a long period. Moreover, with chimeric antigen receptor T-cell 
therapy in the picture, there is no need to subject patients through an ordeal of somewhat contentious second ASCT. However, to adequately answer this question, we would need time for data maturation and possibly a nation-wide observational study.

\section{Future Perspective}

This is indeed an exciting time to treat MM, for physicians and patients alike, because in addition to all the efficacious drugs that have already been approved, there always seems to be a new option with impressive responses on the table. All in all, I think it is fair to deem triplet therapy as the new standard in MM treatment in both newly diagnosed and refractory settings, irrespective of ASCT eligibility. With the introduction of new classes of drugs, such as monoclonal antibodies, we are now being introduced to the results of quadruplet therapy. Tempting as it may be, we should be cautious about declaring quadruplet therapy as the future. Ethnic differences with special regards to safety profiles, sequence of treatment, depth of response, and real-life barriers including the insurance issues need to be addressed. In order to proficiently and safely navigate our patients through the multiple lines of therapy while retaining maximum response, collaborative work is more important than ever. In this regard, I look forward to continued cooperative efforts and dedication from the Korean Multiple Myeloma Working Party and Asian Myeloma Network.

\section{Acknowledgments}

This study was supported by a grant from the Korea Health Technology R\&D Project through the Korea Health Industry Development Institute (KHIDI), funded by the Ministry of Health \& Welfare, Korea (grant No. HI14C2399).

\section{Authors' Disclosures of Potential Conflicts of Interest}

No potential conflicts of interest relevant to this article were reported.

\section{REFERENCES}

1. Mateos MV, Goldschmidt H, San-Miguel J, et al. Carfilzomib in relapsed or refractory multiple myeloma patients with early or late relapse following prior therapy: A subgroup analysis of the randomized phase 3 ASPIRE and ENDEAVOR trials. Hematol Oncol 2018;36:463-70.

2. Stewart AK, Rajkumar SV, Dimopoulos MA, et al. Carfilzomib, lenalidomide, and dexamethasone for relapsed multiple myeloma. N Engl J Med 2015;372:142-52.

3. Dimopoulos MA, Stewart AK, Masszi T, et al. Carfilzomib, lenalidomide, and dexamethasone in patients with relapsed multiple myeloma categorised by age: secondary analysis from the phase 3 ASPIRE study. Br J Haematol 2017;177:404-13.

4. Dimopoulos MA, Stewart AK, Masszi T, et al. Carfilzomiblenalidomide-dexamethasone vs lenalidomide-dexamethasone in relapsed multiple myeloma by previous treatment. Blood Cancer J 2017;7:e554.

5. Al Hamed R, Bazarbachi AH, Malard F, Harousseau JL, Mohty M. Current status of autologous stem cell transplantation for multiple myeloma. Blood Cancer J 2019;9:44.

6. Mohty M, Harousseau JL. Treatment of autologous stem cell transplant-eligible multiple myeloma patients: ten questions and answers. Haematologica 2014;99:408-16.

7. Attal M, Lauwers-Cances V, Hulin C, et al. Lenalidomide, bortezomib, and dexamethasone with transplantation for myeloma. N Engl J Med 2017;376:1311-20.

8. Koreth J, Cutler CS, Djulbegovic B, et al. High-dose therapy with single autologous transplantation versus chemotherapy for newly diagnosed multiple myeloma: A systematic review and meta-analysis of randomized controlled trials. Biol Blood Marrow Transplant 2007;13:183-96.

9. Hagen PA, Stiff P. The role of salvage second autologous hematopoietic cell transplantation in relapsed multiple myeloma. Biol Blood Marrow Transplant 2019;25:e98-107. 